British Journal of Ophthalmology, 1983, 67, 629-634

\title{
Lattice corneal dystrophy: a source of confusion
}

\author{
G. D. STURROCK \\ From the University Eye Clinic, Mittlere Strasse 91, 4056 Basel, Switzerland
}

SUMmary Published descriptions of families with lattice dystrophy of the cornea reveal a much more varied clinical course and a more pleomorphic corneal picture than the rather stereotyped textbook descriptions would suggest. This report describes a family with lattice dystrophy of unusually late onset with retention of useful vision into the 7 th decade. Herpes simplex keratitis was misdiagnosed in 5 members of the family by different ophthalmologists, probably because the typical lattice lines were fine and visible only by retroillumination.

Lattice dystrophy of the cornea is almost invariably described as beginning at the end of the first decade of life or around puberty and progressing to cause severe visual disability by the age of $40 .^{1-6}$ Duke-Elder mentions that the onset of symptoms may be delayed until the third or fourth decades.' All descriptions emphasise the characteristic hyaline, branching lines in the corneal stroma, recognition of which greatly facilitates the correct diagnosis.

A family is described here with lattice dystrophy of unusually late onset in which symptoms never appeared before the fourth decade and in some cases not until the sixth or seventh decades. None of the members of this family realised that they were subject to an inherited corneal disorder. Most of the affected corneae contained only a few rather fine lattice lines which were difficult to see, particularly in the presence of diffuse corneal scarring, which frequently occurs in this dystrophy.

Bilateral herpes simplex keratitis was misdiagnosed in 5 members of the family by different ophthalmologists, probably due to the inconspicuous nature of the lattice lines as well as the negative family history. By the time the proband presented at the Basel Eye Hospital 7 other affected members of the family had already been treated by ophthalmologists (in 2 university eye departments, a regional eye hospital, and at least 7 ophthalmic practices) without the diagnosis of lattice dystrophy having been made.

Correspondence to Dr G. D. Sturrock, Augenspital, Mittlere Strasse 91, 4056 Basel, Switzerland.

\section{Material and methods}

The pedigree of this family containing $\mathbf{5 0}$ members in 4 generations is shown in Fig. 1. Three of the first generation, now all dead, must have been affected, as they reputedly suffered from corneal ulcers and transmitted the dystrophy to their offspring. Thirty-six living members were examined by the author, using a slit-lamp, and 10 were found to have signs of lattice dystrophy. The diagnosis in one of these 10 (case II.10) was confirmed histologically. In case II.6, who had received large, bilateral penetrating grafts, the remaining host cornea in both eyes was normal, but amyloid was demonstrated in the excised corneal discs. The twelfth affected member, who died in 1.972, had been diagnosed as suffering from bilateral herpes simplex keratitis, but must have been affected by the dystrophy which she transmitted to her daughter (case III.8).

\section{Description of afiected members}

CASE II. 1

Female, born 1903 (aged 78).

History. In 1937 the left cornea sustained a fingernail injury, and the resulting erosion took 4 weeks to heal. A spontaneous left corneal erosion occurred in 1968. Visual acuity was recorded as $6 / 6$ in both eyes in 1974. In 1975 the right cornea was scratched by a twig, causing an erosion which finally healed after 8 weeks.

Examination in 1981. Visual acuity: RE 6/18 cc, LE 

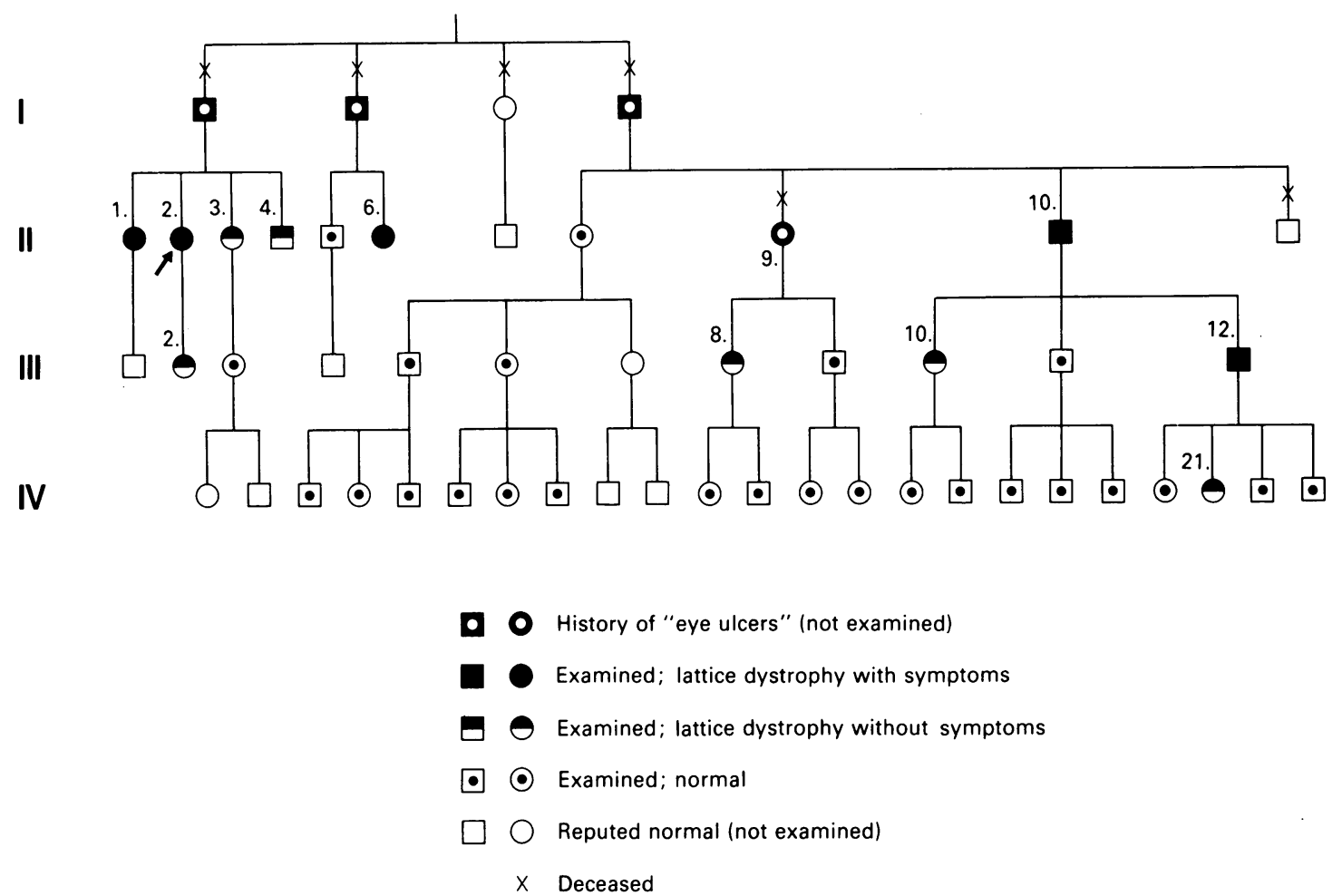

Fig. 1 Pedigree of family with lattice dystrophy of the cornea.

$6 / 18 \mathrm{cc}$. Both corneae appeared normal to the naked eye apart from bilateral arcus senilis. Biomicroscopy revealed a fine superficial stromal haze affecting the central 4 to $5 \mathrm{~mm}$ of each cornea. Within this axial area scattered whitish angular flecks were situated superficially, more numerous in the RE (Fig. 2). Retroillumination from the iris revealed a tangled meshwork of very fine, glassy lines in the central portion of both corneae; only in the right cornea were a few slender lattice lines seen.

CASE II. 2

Proband, female, born 1908 (aged 72).

History. Presented in the casualty department in 1980 with a spontaneous right corneal erosion. No definite history of previous corneal disease.

Examination in 1980. (after healing of the erosion). Visual acuity: RE 6/7.5 cc, LE 6/7.5p cc. The left cornea appeared clear by direct illumination but in the right cornea a discrete, superficial haze was visible slightly below and temporal to the centre. Within this hazy zone was a small, greyish, slightly raised, nodule. Retroillumination revealed a multitude of fine, short lines, like scratches on glass, occupying the axial region of both corneae. A number of tiny stellate or dendritiform lesions were visible at the margins of this mass of lines. A few thin lattice lines, visible only by retroillumination, were seen in the right cornea (Figs. 3a,b).

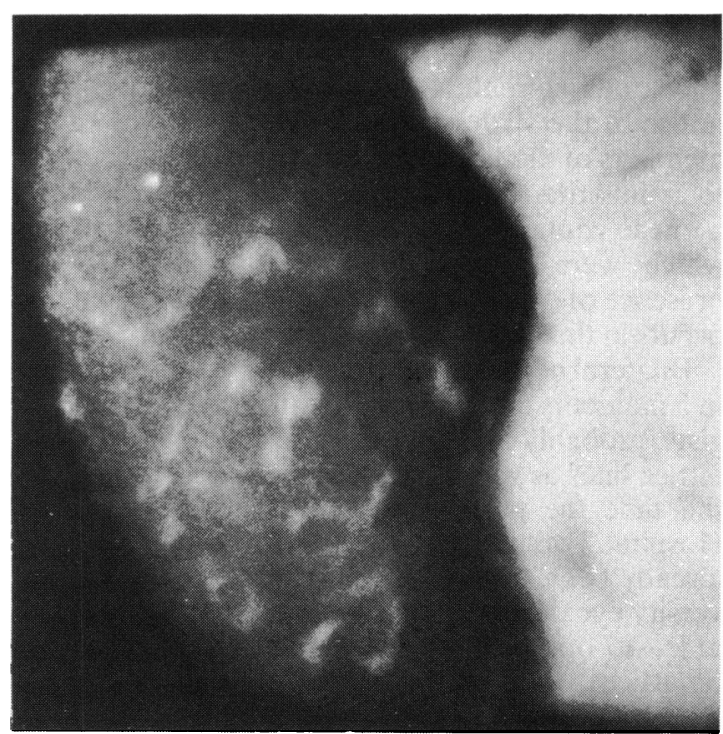

Fig. 2 Case II.1 showing irregular, whitish, subepithelial flecks in the centre of the right cornea. 


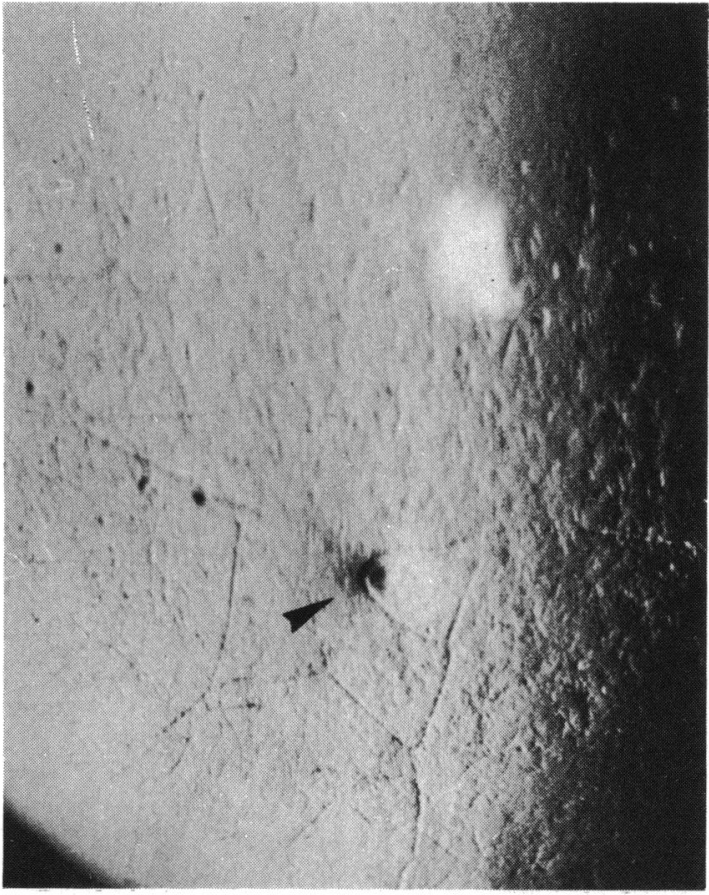

Fig. 3a

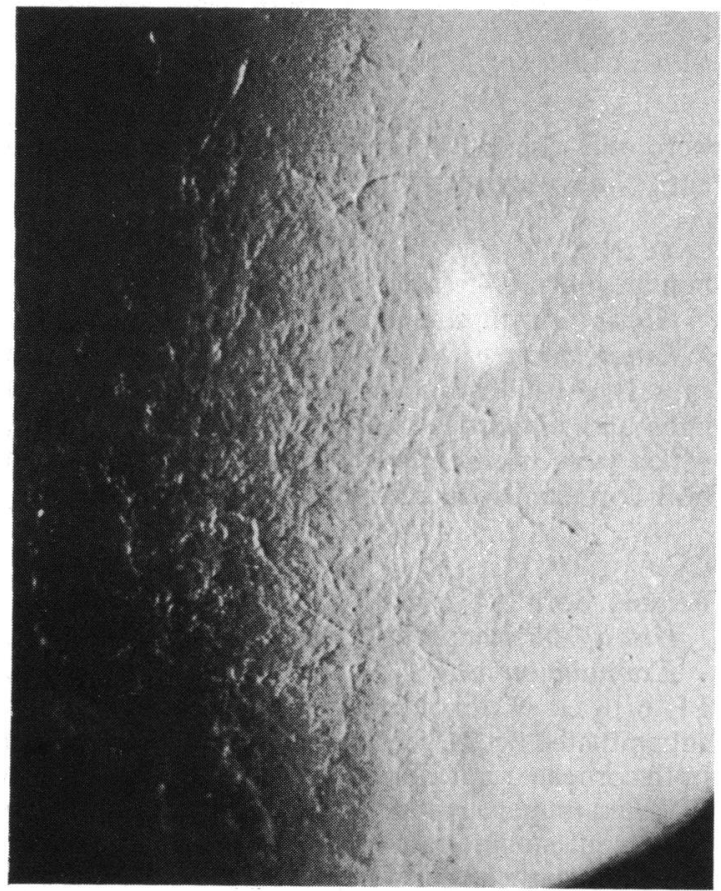

Fig. 3b

Fig. 3(a) Right and (b) left cornea of case II.2 showing short, fine, refractile lines bilaterally, and thin lattice figures and single subepithelial nodule (arrow) in the right cornea.
CASE II. 3

Female, born 1905 (aged 76).

History. A high myope, now almost blind due to bilateral optic atrophy. In 1963 a fine paracentral nebula was recorded in the right cornea and a broad arcus bilaterally. No history of corneal erosions or photophobia.

Examination in 1981. Visual acuity: light perception in both eyes. Bilateral arcus senilis. Biomicroscopy revealed a fine subepithelial haze affecting the central 3.5-4 mm of both corneae. Many very short translucent lines and a few thin lattice figures were visible by retroillumination in each cornea.

CASE II. 4

Male, born 1904 (aged 77).

History. Recent loss of vision due to cataract and macular degeneration. No history of corneal symptoms, but herpes simplex had been diagnosed in 1973 on the basis of scars in the right cornea. Visual acuity then, at the age of 69 , was recorded as RE $6 / 7 \cdot 5$ and LE 6/6.

Examination in 1981. Visual acuity: RE 6/18p, LE $6 / 24 \mathrm{cc}$. The right cornea showed several superficial, slightly elevated, whitish flecks paracentrally. Retroillumination revealed fine translucent lines bilaterally, apart from which the corneal stroma was basically clear.

\section{CASE II. 6}

Female, born 1911 (aged 70).

History. From 1960, aged 49, she suffered recurrent attacks of corneal erosions affecting both eyes. They were diagnosed as herpes simplex keratitis and treated by repeated corneal abrasions and later with antiviral agents. Increasing corneal scarring had reduced the vision to RE 6/18 and LE $6 / 9$ by 1979 when a right penetrating graft was performed, followed by a similar operation on the left eye in 1980. Reis-Bückler's dystrophy was diagnosed histologically. However, re- examination of the corneal discs one year later in Basel with Congo red stain revealed the presence of amyloid, confirming the diagnosis of lattice dystrophy.

Examination in 1981. Visual acuity: RE 6/9 cc, LE CF. Bilateral clear grafts with no evidence of lattice dystrophy in the remaining cornea. Arcus senilis (left cataract).

CASE II. 9

Female (1908-72).

History. Treated by a private ophthalmologist for a left corneal erosion in 1965. Three years later, aged 60 , bilateral herpes simplex keratitis was diagnosed. Visual acuity was then recorded as RE 6/7.5 and LE 
6/9. Died in 1972 and was never examined by the author. Her daughter (case III.8) has lattice dystrophy.

CASE II. 10

Male, born 1905 (aged 76).

History. Left corneal erosion in 1939. Right corneal erosion in 1945, diagnosed as herpetic keratitis. He was admitted to hospital and treated by corneal abrasion and application of iodine. After healing, visual acuity in the RE was $6 / 12$ due to a central macula, and 6/5 in the LE. Between 1946 and 1956 he was treated for recurrent attacks of 'herpes' affecting both eyes. By 1963 corrected visual acuities were RE 6/18 and LE 6/9+ . A right lamellar graft was performed; no definite histological diagnosis could be made. By 1975 vision in the LE was $6 / 30$ and a left lamellar graft was carried out. In 1981 re-examination of the corneal material obtained in 1963 showed the presence of amyloid when stained with Congo red.

Examination in 1981. Visual acuity: RE CF at $2 \mathrm{~m}$, LE 6/12 cc. Prominent arcus senilis in both corneae with apparent reduplication in the upper periphery of the left graft (Fig. 4), which was otherwise clear. The right graft showed areas of white, reticular, subepithelial scarring. Delicate lattice lines were visible in the peripheral host cornea and deep to the lamellar graft.

CASE III. 2

Female, born 1941 (aged 40).

History. No symptoms of corneal disease.

Examination in 1981. Visual acuity: $6 / 6$ in both eyes. Both corneae contained numerous tiny, translucent, stellate or dendritiform lesions which

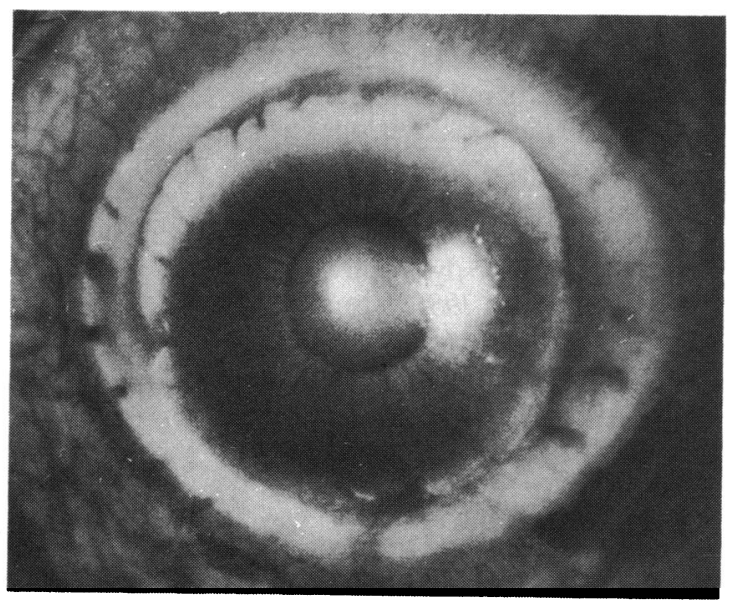

Fig. 4 Case II.10 showing reduplication of arcus senilis in upper margin of lamellar graft.

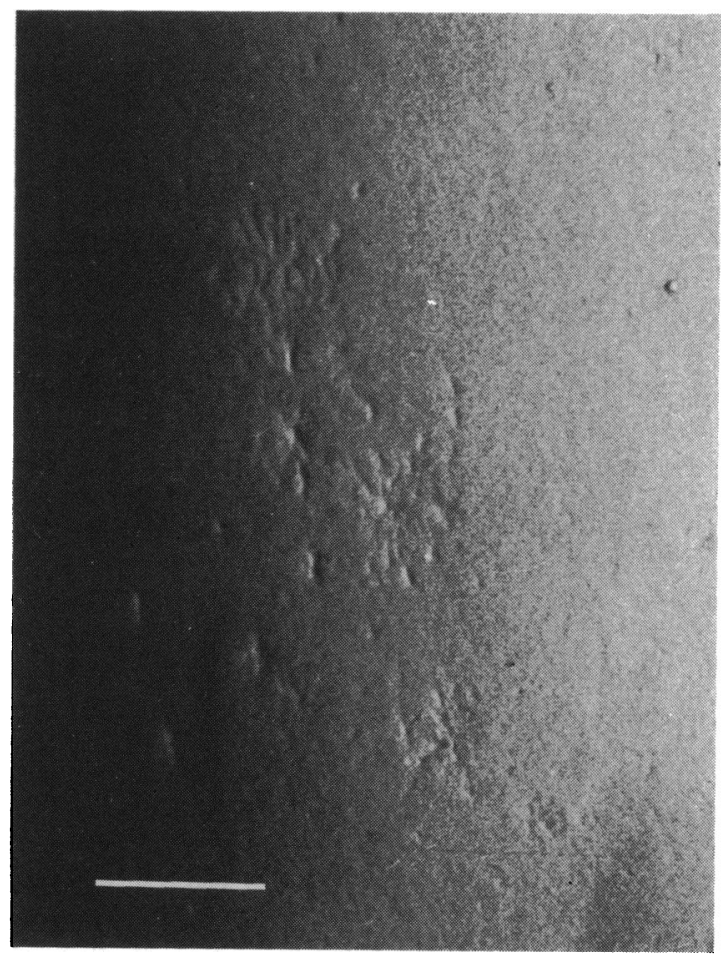

Fig. 5 Case III.2 showing tiny, translucent, dendritiform lesions, visible only by retroillumination. Bar $=500 \mu \mathrm{m}$.

were only visible by retroillumination (Fig. 5). No lattice lines were seen.

CASE III.8.

Female, born 1945 (aged 36).

History. Asymptomatic.

Examination in 1981. Visual acuity: $6 / 6$ in both eyes. By retroillumination, many very fine translucent lines and numerous tiny, hyaline, stellate lesions, which were more or less confluent, were visible in both corneae. No lattice figures were found.

CASE III. 10

Female, born 1943 (aged 38).

History. No symptoms of corneal dystrophy.

Examination in 1981. Visual acuity: RE 6/18+ cc, LE 6/18 cc. A few irregularly shaped, hazy white, subepithelial flecks were visible in the axial region of both corneae, right more than left. Retroillumination revealed numerous very fine, short, translucent lines (confluent stellate lesions) in both comeae. Typical branching lattice lines were not seen.

CASE III.12

Male, born 1935 (aged 46).

History. Intermittent attacks of pain affecting first 


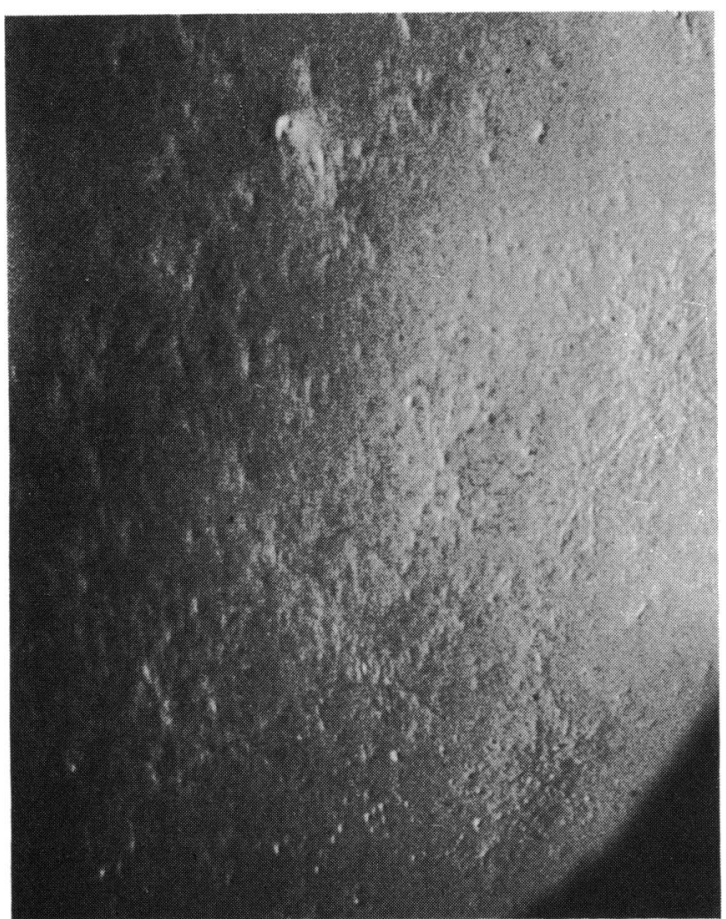

Fig. 6 Case III.12 showing confluent stellate and dendritiform lesions. Retroillumination.

the left then the right eye for the past 18 months. Diagnosed and treated as herpes simplex keratitis.

Examination in 1981. Visual acuity: 6/6 in both eyes. Both corneae contained about a dozen irregular whitish, subepithelial opacities distributed in the pupillary zone. Numerous small translucent stellate lesions, almost confluent in places, were visible by retroillumination in the stroma of both corneae (Fig. 6). A single classical lattice line was found in the left cornea.

\section{CASE IV. 21}

Female, born 1966 (aged 15).

History. No eye symptoms.

Examination in 1981. Visual acuity: $6 / 6$ in both eyes. The left cornea contained 3 tiny, translucent, stellate lesions in the stroma, visible only by retroillumination. The right cornea was normal.

\section{Discussion}

The autosomal dominant inheritance of the corneal condition described in thisfamily, the presence of fine lattice-like lines in many of the affected corneae, and the histological demonstration of amyloid in 2 cases confirm the diagnosis of lattice dystrophy. However, the late onset and generally benign course hardly conform to the rather stereotyped description of this dystrophy found in most textbooks. Failure to appreciate the striking variation in age of onset, symptoms, and corneal findings which different families with lattice dystrophy may show can lead to misdiagnosis, as occurred in the present family.

The oft repeated statement that lattice dystrophy becomes manifest between the first and second decades of life is not borne out by a study of published pedigrees. While affected members of the same family tend to develop symptoms at approximately the same stage of life, within a period spanning 10 to 15 years, there is considerable variation between different families in the age at which symptoms first appear. Rarely, lattice dystrophy may produce symptoms as early as the first year of life, ${ }^{8}$ it may start during childhood, ${ }^{910}$ between the ages of 15 to 25 years, ${ }^{11}$ during the third ${ }^{12}$ or fourth decades, ${ }^{13}$ or as late as the fifth and sixth decades. ${ }^{9}$ The age at onset of symptoms in the present family ranged between 40 to as late as 72 years, apart from case II.10, who suffered a single erosion aged 34 and then remained asymptomatic for the next 6 years.

The characteristic symptoms of lattice dystrophy are recurrent, painful, corneal erosions, photophobia, and progressive loss of vision. The attacks of pain may precede the visual loss, occur at irregular intervals, and tend to become less frequent as the disease progresses. However, it is quite unusual for a patient with lattice dystrophy not to experience corneal erosions at some stage. Yet only 6 of the 12 patients in the family described here had experienced spontaneous erosions, while the other 6 affected members gave no history at all of such attacks.

Progression of the corneal opacities and scarring leads ultimately to visual incapacity in most patients some time between the fourth and sixth decades. The average age of 23 patients receiving corneal grafts for lattice dystrophy was 47 years, with a range of 20 to 76 years. ${ }^{14}$ Preservation of useful vision after the age of 60 would appear, from a review of published cases, to be most unusual. However, 4 members of the present family retained a visual acuity of $6 / 9$ or better in both eyes when aged $60,69,71$, and 72 respectively. Two other members of the family, also in the second generation, both had visual acuities of 6/9 in one eye and 6/18 in the other when aged 58 .

The typical branching lattice figures in the corneal stroma, after which this dystrophy is named, are so characteristic that, once recognised, a confident diagnosis can be made. However, several other types of corneal opacity are regularly seen in this dystrophy and in the early stages may precede the appearance of lattice figures and in the late stages may overshadow or hide these classical lesions. At least 5 distinct types of corneal opacity comprising minute, stellate lesions; 
fine, short lines; branching lattice figures; whitish subepithelial flecks and stroma clouding were seen in members of the present family.

Tiny, translucent, stellate or dendritiform lesions, initially visible only by transillumination but later becoming faintly greyish, were the earliest changes seen. Very fine, short lines resembling scratches on glass and visible only by retroillumination appeared when the stellate lesions increased in number to become confluent. Classical branching lattice figures were inconspicuous and indeed could not be identified in every affected cornea. When present they were rather fine, few in number, and, like the little stars and short lines, could be seen only by retroillumination. Angular whitish flecks, between 6 and 12 in number and situated superficially, were visible in 4 affected members. A cloudy haze involving the anterior stroma in the axial region of the cornea was seen in 3 elderly patients. The flecks and cloudy haze were visible in direct illumination.

The single, subepithelial lesion in the right cornea of case II.2 (Fig. 3a), faintly greyish in direct illumination and 'nodular' by retroillumination, probably corresponds to the transparent nodules described in family 12 (cases 1,2 , and 5) by Bücklers. ${ }^{12}$

The spectrum of corneal changes described here illustrates the extraordinarily pleomorphic nature of lattice dystrophy which previous authors have also noted. ${ }^{12} 1516$ If the diagnosis of lattice dystrophy is restricted solely to patients with prominent lattice figures, then clearly some cases will be overlooked or misdiagnosed, as occurred in 6 members of the present family. Thus case II.1, who presented in 1975 with a traumatic erosion, was examined by at least 5 different ophthalmologists none of whom detected the presence of lattice dystrophy. The 5 patients in whom herpes simplex keratitis was misdiagnosed were less fortunate, particularly cases II.6 and II.10, both of whom were treated by repeated abrasions and, in case II.10, by iodine applications. As these were the only 2 members of this family submitted to corneal grafting, it is quite possible that the misconceived treatment which they received precipitated the dense superficial corneal scarring for which surgery was ultimately required.
This family demonstrates very clearly the importance of using retroillumination routinely in the course of corneal examination with the slit-lamp. Failure to do so must have been responsible for the diagnostic confusion described in this report, since characteristic glassy lines were present in all except the 2 youngest members of the family but were detectable only by retroillumination. Although 2 types of corneal opacity were visible by direct illumination, namely, the irregular, whitish, subepithelial flecks and the superficial, central stromal haze, neither is sufficiently specific at first sight to suggest the diagnosis of lattice dystrophy.

Professor Daicker kindly performed the histochemical staining for amyloid in cases II.6 and II.10.

\section{References}

1 Duane TD, Jaeger EA. Clinical ophthalmology. Philadelphia: Harper and Row, 1982: 4: 20-3.

2 François J, Hollwich F. Augenheilkunde in Klinik und Praxis. Stuttgart, New York: Thieme, 1981: 2: 92-5.

3 Grayson M. Diseases of the cornea. St Louis: Mosby, 1979: 216-221.

4 Donaldson DD. Atlas of external diseases of the eye. St Louis: Mosby, 1971: 3: 150-8.

5 Peyman GA, Sanders DR, Goldberg MF. Principles and practice of ophthalmology. Philadelphia: Saunders, 1980: 1: 428-31.

6 Thomas CI. The cornea. Springfield: Thomas, 1955: 289-90.

7 Duke-Elder S. System of ophthalmology. London: Kimpton, 1965; 8 (2): 933-9.

8 Stansbury CF. Lattice type of hereditary corneal degeneration. Arch Ophthalmol 1948; 40: 189-217.

9 Dark AJ, Thomson DS. Lattice dystrophy of the cornea: a clinical and microscopical study. Br J Ophthalmol 1960; 44: 257-79.

10 Colvin JL, Blyth $\mathrm{H}$. Lattice dystrophy of the cornea: occurrence in 4 generations of a Yorkshire family. Br J Ophthalmol 1962; 46: 549-53.

11 Hesse E. Beitrag zum Beginn und Erblichkeit der gittrigen Hornhautdystrophie. Albrecht von Graefes Arch Klin Ophthalmol 1939; $141: 1-19$.

12 Bücklers M. Die erblichen Hornhautdystrophien. Dystrophiae corneae hereditariae. Klin Monatsbl Augenheilkd 1938; suppl 3.

13 Ramsay RM. Familial corneal dystrophy lattice type. Trans Am Ophthalmol Soc 1957; 55: 701-39.

14 Lanier DJ, Fine M, Togni B. Lattice corneal dystrophy. Arch Ophthalmol 1976; 94: 921-4.

15 Dubord PJ, Krachmer JH. Diagnosis of early lattice corneal dystrophy. Arch Ophthalmol 1982; 100: 788-90.

16 Fuchs A. Zur Kenntnis der gittrigen Hornhautentartung. $Z$ Augenheilkd 1926; 57: 159-86. 